\title{
Farmacología de los azoles
}

\author{
José Ramón Azanza, Emilio García-Quetglas y Belén Sádaba \\ Servicio de Farmacología Clínica, Clínica Universitaria de Navarra. Facultad de Medicina Universidad de \\ Navarra, Pamplona, Navarra \\ Resumen Los azoles presentan características farmacocinéticas diferenciadas, con una \\ absorción oral completa de voriconazol, menor de fluconazol y menos \\ importante y que aumenta con alimentos en el caso de posaconazol e \\ itraconazol. Todos presentan una distribución tisular elevada, que se \\ manifiesta por concentraciones plasmáticas muy reducidas, especialmente en \\ el caso de posaconazol e itraconazol. Estos dos últimos fármacos presentan \\ una fracción libre reducida al circular en plasma unidos a proteínas en \\ proporción elevada. La eliminación es en todos los casos a través de \\ metabolismo con intervención de diversas isoenzimas CYP450, a las que son \\ capaces de inhibir, lo que condiciona una farmacocinética no lineal y un \\ elevado riesgo de interacciones con otros fármacos. Es posible que el \\ parámetro que mejor defina la eficacia sea el AUIC con un valor óptimo de 20. \\ Si esta circunstancia se concreta, debe valorarse el punto de corte para la \\ sensibilidad puesto que algunos de estos fármacos pueden tener dificultades \\ para alcanzar este valor. \\ Palabras clave Farmacología, Posaconazol, Itraconazol, Fluconazol, Voriconazol

\section{Pharmacology of azoles} \\ Summary Azole antifungals have different pharmacokinetic characteristics: complete oral \\ absorption for Voriconazole, and to a lesser extent for fluconazole. \\ The absorption of posaconazole and itraconazole increases with food intake. \\ All of them have high tissue distribution with low plasma concentrations, \\ especially low in the case of posaconazole and itraconazole. Posaconazole \\ and itraconazole have high plasmatic protein binding and consequently both \\ have a very low free fraction. Elimination of azole antifungals is through a \\ metabolic pathway with CYP450 isoenzymes, and has a non linear \\ pharmacokinetics with a high risk of interation with other drugs since azoles \\ have the ability of CYP450 isoenzymes inhibition. Possibly the parameter that \\ defines more precisely their efficacy is AUIC with an optimum value near 20, \\ although cut-off values must be defined since some azoles may have difficulty \\ to reach this value.
}

Key words Pharmacology, Posaconazole, Itraconazole, Fluconazole, Voriconazole

Los azoles forman una familia heterogénea de fármacos que comparten la presencia de un anillo azólico central y con ello el mecanismo de acción antifúngico, que consiste en la inhibición de la síntesis del ergosterol. Al mismo tiempo los cuatro azoles de interés en el tratamiento de las infecciones sistémicas: fluconazol, itraconazol, voriconazol y posaconazol, presentan diferencias importantes en su estructura, con similitud entre ellos. Estas diferencias en la estructura generan comportamien-

Dirección para correspondencia:

Dr. José Ramón Azanza

Servicio de Farmacología Clínica

Clínica Universitaria de Navarra

Avda. Pío XII s/n

31008 - Pamplona

Tel.: (+34) 948296695

Fax: (+34) 948296500

E-mail: jrazanza@unav.es

(ㄷ)2007 Revista Iberoamericana de Micología

Apdo. 699, E-48080 Bilbao (Spain)
$1130-1406 / 01 / 10.00 €$ tos farmacocinéticos distintos que tienen repercusiones notables en el manejo práctico de estos fármacos, a los que se hará referencia en este trabajo, referido fundamentalmente a itraconazol, posaconazol y voriconazol.

\section{Características farmacocinéticas}

En la tabla 1 se describen los parámetros farmacocinéticos más relevantes de cada uno de los fármacos a los que se hará referencia a lo largo de este apartado.

La absorción oral más elevada se obtiene con voriconazol, fármaco que administrado en ayunas alcanza una biodisponibilidad próxima al 100\% [25-27], cifra que es inferior a su vez en el caso de itraconazol en solución (70\%) [21], y que se reduce cuando este fármaco se administra en forma de cápsulas, hasta situarse en el 55\%, cifra que es inferior cuando este fármaco se administra junto con una suspensión de alcalinos [18]. La biodisponibilidad de posaconazol, solo comercializado en solución para uso por vía oral, se sitúa en torno al 60\% [10]. La administración con alimentos mejora la biodisponibilidad de estos dos últimos fármacos [10,30]. 
Tabla 1. Parámetros farmacocinéticos de los azoles $[8,14,17,21,24-27,31]$

\begin{tabular}{lcccc}
\hline & Fluconazol & Itraconazol & Posaconazol & Voriconazol \\
\hline Dosis /vía & $400 / \mathrm{IV}$ & $100 / \mathrm{IV}$ & $400 / \mathrm{PO}$ & $200 / \mathrm{PO}$ \\
$\mathrm{C}_{\max }(\mathrm{mg} / \mathrm{l})$ & 18,9 & 1,9 & 0,8 & 1,7 \\
$\mathrm{t}_{\max }(\mathrm{h})$ & 1,5 & $3-5$ & 3.4 & $1-2$ \\
$\mathrm{Vd}_{\mathrm{d}}(\mathrm{l} / \mathrm{kg})$ & 0,50 & 10 & $7-20$ & $2-5$ \\
$\mathrm{FP}(\%)$ & 12 & 99 & 98 & 58 \\
$\mathrm{U}(\%)$ & 60 & $<5$ & $<5$ & $<5$ \\
$\mathrm{t}_{1 / 2}(\mathrm{~h})$ & 30 & $30-40$ & $12-24$ & $6-9$ \\
$\mathrm{AUC}(\mathrm{mgxh} / \mathrm{l})$ & 350 & $3-6$ & $5-8$ & $14-43$ \\
$\mathrm{~F}(\%)$ & 80 & $55-70^{1}$ & $60-80$ & 95 \\
\hline
\end{tabular}

$\mathrm{C}_{\max }$ : concentración plasmática máxima.

tmax: tiempo en el que se alcanza la concentración plasmática máxima.

Vd: volumen de distribución.

FP: fijación a proteínas.

U: fracción eliminada activa por la orina.

t 12 : semivida de eliminación.

AUC: área bajo la curva.

F: biodisponibilidad por vía oral.

: cápsulas/solución.

La escasa solubilidad de posaconazol se ha puesto en relación con una circunstancia diferencial y curiosa puesto que resulta poco frecuente, y que se concreta en los parámetros biodisponibilidad (expresada por el área bajo la curva, AUC) y concentración plasmática máxima $\left(\mathrm{C}_{\max }\right)$, parámetros cuyo valor se reduce de forma considerable, conforme se aumenta la cantidad administrada en cada dosis $[8,13,14]$. Por ello se ha propuesto utilizar dosis de $400 \mathrm{mg}$ cada $12 \mathrm{~h}$, si el fármaco se ingiere con alimentos, ó $200 \mathrm{mg}$ cada $6 \mathrm{~h}$ si se ingiere en ayunas, dosis esta que alcanza mayor biodisponibilidad que los $400 \mathrm{mg}$.

El elevado volumen de distribución de todos los azoles unido, en los casos mencionados, a la biodisponibilidad oral moderada, justifican que las concentraciones plasmáticas alcanzadas inicialmente, sean reducidas, hasta el punto de que en el caso de alguno de ellos, como posaconazol, la $\mathrm{C}_{\max }$ alcanzada con el rango de dosis propuesta no supera los $0,9 \mathrm{mg} / \mathrm{l}$, cifra que es inferior a la del punto de corte propuesto para definir la sensibilidad a este fármaco $(1 \mathrm{mg} / \mathrm{l})$.

Este es el motivo que justifica que se recomiende administrar dosis de carga con voriconazol (en las dos vías de administración) y con itraconazol (solo la vía IV), pero sorprendentemente no se recomiende para la vía oral de este último ni para posaconazol, lo que potencialmente podría llegar a retrasar el efecto farmacológico en la sangre, con posible trascendencia, al menos en teoría, en el tratamiento de candidemias.

Una característica que puede tener gran interés es la necesidad de circular en el plasma fijado a proteínas en elevada proporción que, en los casos de posaconazol [9] e itraconazol superan el $90 \%$ del fármaco circulante, mientras que para voriconazol este porcentaje se sitúa en el $58 \%$. Considerando la reducida entidad de las concentraciones plasmáticas y el dato señalado de la fijación a proteínas, es destacable que la concentración plasmática libre resulte muy baja, especialmente en el caso de posaconazol $[8,14,31]$ e itraconazol que, por otra parte, son los fármacos que presentan menor biodisponibilidad.

Todos los azoles tienen un volumen de distribución muy elevado que supera, con creces, el contenido de agua corporal que señala de forma clara la afinidad tisular de estos fármacos, y justifica la escasa presencia en plasma $[8,14,17,31]$. Voriconazol, de forma característica, y que probablemente comparte con los restantes azoles, alcanza una concentración intracelular elevada en polimorfonucleares humanos con una penetración rápida, no saturable e independiente de las condiciones ambientales [5]. No existe demasiada información sobre las concentraciones tisulares y la existente se ha centrado en la distribución de estos fármacos en el sistema nervioso central (SNC) y algunos otros tejidos de interés. En el primero, SNC, se ha descrito la consecución de concentraciones adecuadas $[11,12,19]$ o reducidas [11] de voriconazol y de menor entidad con itraconazol y fluconazol [4].

Se ha estudiado la concentración en los humores vítreo y acuoso del ojo que en el caso de voriconazol resulta el 38,1 y 53\%, respectivamente, de la plasmática, y que son superiores a las necesarias para inhibir la multiplicación de cualquiera de los hongos y de las levaduras sensibles que producen la infecciones oculares [15]. Se ha descrito que voriconazol alcanza concentraciones adecuadas en líquido peritoneal cuando se administra a la dosis convencional por vía oral en pacientes con diálisis peritoneal [23].

También se ha estudiado la difusión en el líquido de revestimiento epitelial intrapulmonar alcanzándose, en el caso de voriconazol, una concentración que supera de media a la plasmática unas 11 veces [6]. Con itraconazol y tras la administración oral de $200 \mathrm{mg}$ cada 12 h en dosis múltiple, se observaron resultados similares que incluyen concentraciones elevadas dentro de las células alveolares [7].

La eliminación de los azoles se realiza a través de metabolismo en el que intervienen algunas de las isoenzimas del sistema microsomal CYP450. Es decir, la excreción renal, si se excluye a fluconazol, no interviene en la eliminación de los azoles, lo que supone la ausencia de acumulación de estos fármacos cuando se administran a pacientes con disfunción renal [9]. En los casos de las formulaciones intravenosas de voriconazol e itraconazol, existe riesgo de acumulación de uno de los excipientes utilizado en la solubilización; una ciclodextrina. Se trata de azúcares complejos que son eliminados del organismo a través de la orina tras filtración glomerular y que por consiguiente, en el caso de que la función renal esté deteriorada, sufren acumulación. En la actualidad aún se desconocen las consecuencias que esta acumulación supone para la tolerabilidad de la ciclodextrina, aunque existen algunas experiencias en las que no se produjo ningún signo de intolerancia [32]. Se recomienda evitar la administración de estos fármacos en su formulación intravenosa, en pacientes con disfunción renal moderada y severa (CLCr $<30-50 \mathrm{ml} / \mathrm{min}$ ), mientras que no existe restricción alguna en la utilización de las formulaciones orales.

Voriconazol no es dializado por lo que no se precisa ajustar la dosis cuando se administra por vía oral [23], característica que comparte con posaconazol [9] e itraconazol [20], habiéndose descrito que la hemodiálisis es eficaz para eliminar del organismo la ciclodextrina utilizada como excipiente de la formulación intravenosa de este fármaco [20].

La intervención del sistema microsomal hepático en el metabolismo de los azoles obliga a valorar la repercusión que la alteración de la función hepática puede tener sobre la velocidad de eliminación de estos fármacos. Los estudios realizados parecen aconsejar utilizar los fármacos con precaución en pacientes con Child-Pugh $\mathrm{B}$ y $\mathrm{C}$, reduciendo la dosis de mantenimiento a la mitad, especialmente en la administración intravenosa. Además, la implicación de este complejo sistema enzimático obliga a valorar la posibilidad de que se produzcan interacciones con potencial riesgo, sustentadas en este caso, en la inhibición de las isoenzimas que participan en su metabolismo. Itraconazol, voriconazol [17] y posaconazol son capaces 
Tabla 2. Interacciones de los azoles [3]

Disminución de la concentración del azol

\begin{tabular}{|c|c|c|c|c|c|}
\hline \multirow[t]{2}{*}{ Efecto/Fármaco } & \multicolumn{4}{|c|}{ Azol(es) afectados } & \\
\hline & itraconazol & fluconazol & posaconazol & voriconazol & \\
\hline \multicolumn{6}{|c|}{ Disminución de la absorción: } \\
\hline Antiácidos & $\mathrm{x}$ & $\mathrm{x}$ & & & \\
\hline Antagonistas $\mathrm{H} 2$ & $x$ & $x$ & $x$ & $x$ & \\
\hline Sucralfato & $x$ & & & & \\
\hline Omeprazol & $x$ & & & & \\
\hline Didanosina & $x$ & & & & \\
\hline Gemfibrozilo & $x$ & & & & \\
\hline \multicolumn{6}{|c|}{ Aumento del metabolismo del azol: } \\
\hline Rifampicina & $x$ & $x$ & $x$ & $x$ & \\
\hline Fenitoína & $x$ & & & & \\
\hline Carbamazepina & $x$ & & $\mathrm{x}$ & $x$ & \\
\hline Fenobarbital & $x$ & & $x$ & $x$ & \\
\hline Nevirapina & & & & $x$ & \\
\hline Efavirenz & & & & $x$ & \\
\hline \multicolumn{6}{|c|}{ Aumento de concentraciones del fármaco coadministrado: } \\
\hline Fármaco & itraconazol & fluconazol & posaconazol & voriconazol & Consecuencias \\
\hline Alfentanilo & $\mathrm{x}$ & $x$ & & & Sedación \\
\hline Alprazolam & $x$ & $x$ & $x$ & $x$ & Sedación \\
\hline Amprenavir & & & & & Riesgo de intoxicación \\
\hline Anticoagulantes orales & $x$ & $x$ & $x$ & $x$ & Riesgo de hemorragia \\
\hline Aripiprazol & $x$ & & & & Riesgo de intoxicación \\
\hline Astemizol & $x$ & & $x$ & $x$ & Prolongación QT \\
\hline Atorvastatina & $x$ & & & $x$ & Riesgo de rabdomiolisis \\
\hline Budesonida & $x$ & & & $x$ & Riesgo de intoxicación \\
\hline Bupivacaina & $x$ & & & & Riesgo de intoxicación \\
\hline Buspirona & $x$ & & & & Riesgo de intoxicación \\
\hline Carbamazepina & & $x$ & & & Riesgo de intoxicación \\
\hline Celecoxib & & $x$ & & & Riesgo de intoxicación \\
\hline Ciclosporina & $x$ & $x$ & $x$ & $x$ & Riesgo de intoxicación \\
\hline Citarabina & $x$ & & & & Riesgo de intoxicación \\
\hline Clordiacepóxido & & & & & Sedación \\
\hline Dexametasona & $x$ & & & $x$ & Riesgo de intoxicación \\
\hline Digoxina & $x$ & & & $x$ & Riesgo de intoxicación \\
\hline Disoporamida & $\hat{x}$ & & $x$ & $\hat{x}$ & Arritmias \\
\hline Ergotamínicos & $x$ & & $x$ & $x$ & Riesgo de intoxicación \\
\hline Everolimus & $x$ & & & & Riesgo de intoxicación \\
\hline Felodipino & $x$ & & $x$ & & Edema \\
\hline Feniotína & $x$ & $x$ & $x$ & $x$ & Riesgo de intoxicación \\
\hline Fentanilo & & $x$ & & & Sedación \\
\hline Fluoxetina & $x$ & & & & Riesgo de intoxicación \\
\hline Fluvastatina & & $x$ & & & Riesgo de rabdomiolisis \\
\hline Imipramina & $x$ & & & & Riesgo de intoxicación \\
\hline Indinavir & $x$ & & & & Riesgo de intoxicación \\
\hline Irbesartan & & & & & Riesgo de intoxicación \\
\hline Lidocaína & $x$ & & $x$ & $x$ & Arritmias \\
\hline Loratidina & $x$ & $x$ & $x$ & $x$ & Riesgo de prolongación QT \\
\hline Losartan & & $\mathrm{x}$ & & & Hipotensión \\
\hline Lovastatina & $\mathrm{x}$ & $x$ & & $x$ & Riesgo de rabdomiolisis \\
\hline Metilprednisolona & $x$ & & & $x$ & Riesgo de intoxicación \\
\hline Midazolan & $x$ & $x$ & $x$ & $x$ & Sedación \\
\hline Nevirapina & & $x$ & & & Riesgo de intoxicación \\
\hline Nifedipino & $x$ & & $x$ & & Edema \\
\hline Nortriptilina & & $x$ & & & Sedación, arritmias \\
\hline Pimozida & $x$ & & $x$ & $x$ & Prolongación QT \\
\hline Pravastatina & & & & & Riesgo de rabdomiolisis \\
\hline Quinidina & $x$ & & $x$ & $x$ & Arritmias \\
\hline Reboxetina & $x$ & & & & Riesgo de intoxicación \\
\hline Rifabutina & & $x$ & & & Uveitis \\
\hline Ritonavir & & & & & Riesgo de intoxicación \\
\hline Ritonavir & $x$ & $x$ & & & Riesgo de intoxicación \\
\hline Saquinavir & & $x$ & & & Riesgo de intoxicación \\
\hline Simvastatina & $x$ & $x$ & & $x$ & Riesgo de rabdomiolisis \\
\hline Sirolimus & $x$ & & $x$ & $x$ & Riesgo de intoxicación \\
\hline Sulfonilureas & $x$ & $x$ & $x$ & $x$ & Riesgo de hipoglucemia \\
\hline Tacrolimus & $x$ & $x$ & $x$ & $x$ & Riesgo de intoxicación \\
\hline Terfenadina & $x$ & & $x$ & $x$ & Prolongación QT \\
\hline Trazodona & $x$ & & & & Riesgo de intoxicación \\
\hline Triazolam & $x$ & $x$ & $x$ & $x$ & Sedación \\
\hline Triptanes & $x$ & & & & Riesgo de intoxicación \\
\hline Venlafaxina & $x$ & & & & Riesgo de intoxicación \\
\hline Vincristina & $x$ & & & & Riesgo de intoxicación \\
\hline Vinorelbina & $x$ & & & & Riesgo de intoxicación \\
\hline Zidovudina & & $x$ & & & Riesgo de intoxicación \\
\hline
\end{tabular}


de inhibir con gran actividad, la CYP3A4, además, voriconazol inhibe la CYP2C9 y la CYP2C19 [22]. Esta característica se convierte en uno de los principales inconvenientes del manejo de los azoles y supone dos consecuencias de importancia: una farmacocinética no lineal [8], y el riesgo de interacciones con consecuencias relevantes para el paciente.

La farmacocinética no lineal de los azoles se caracteriza por un aumento de las concentraciones conforme progresa el tratamiento. Tiene su expresión en el aumento de la semivida de eliminación, como consecuencia de la reducción del aclaramiento que tiene su origen en la inhibición de la actividad de las enzimas que metabolizan al fármaco. Por consiguiente, los efectos beneficiosos y adversos que dependen directamente de las concentraciones que alcanzan los fármacos, pueden ser más evidentes en el tiempo.

En referencia a las interacciones, la implicación de estos fármacos es evidente ya que se encuentran entre los que presentan mayor capacidad inhibitoria del metabolismo de otros fármacos. De una manera global resulta sencillo sospechar los fármacos que pueden ser diana de la interacción, basta con revisar si entre las enzimas encargadas de su metabolismo se encuentra alguna de las inhibidas por los azoles. Si esto sucede y además la enzima tiene especial protagonismo en la eliminación del fármaco, existirá riesgo elevado de que se vea reducido su aclaramiento, con el correspondiente aumento de la semivida de eliminación y el riesgo de acumulación, si no se reduce la dosis. En la tabla 2 se describen, de forma resumida, las diferentes interacciones en las que se encuentran implicados los azoles y sus consecuencias. La intensidad de la inhibición es llamativa [29]. A modo de ejemplo se ha señalado que se precisa reducir la dosis de los diferentes inmunosupresores en presencia de azoles en porcentajes variables aunque muy elevados, superiores al 60\% [28].
Se ha estudiado el comportamiento farmacocinético de algunos de estos fármacos en niños [16]. Voriconazol muestra un perfil de eliminación dependiente de la edad, caracterizado por un aumento del aclaramiento y reducción de la semivida de eliminación de origen no aclarado, pero relacionado probablemente con diferencias en la actividad de las CYP450, y que exige la administración de dosis superiores a las utilizadas habitualmente en adultos [33].

Las interrelaciones entre la farmacocinética y la farmacodinamia de los antimicrobianos están adquiriendo cierta trascendencia a la hora de comparar los fármacos y de establecer modelos de eficacia/tolerancia. En el caso de los azoles no se dispone de información clínica relevante, aunque en los modelos de animales vivos, es la relación AUC con la CMI (AUIC), el parámetro que mejor predice la eficacia de estos antifúngicos. Los estudios realizados parecen señalar que el valor óptimo del AUIC se sitúa en 20 [1,2]. La comparación de las AUC (Tabla1) permite comprobar las diferencias en el valor AUC/CMI de los diferentes azoles si se utiliza en el cálculo el mismo valor para la CMI. Si además se utiliza el valor de AUC correspondiente a la fracción libre, las diferencias son aún más importantes situando a voriconazol en cifras muy superiores a las de los restantes fármacos. La información referente al valor adecuado de la AUIC es real, y parece evidente que algunos fármacos pueden tener dificultades cuando deban de ser eficaces en el tratamiento de infecciones producidas por cepas que presenten una CMI próxima a $1 \mathrm{mg} / \mathrm{l}$. 
1. Andes D, Marchillo K, Stamstad T, Conklin R. In vivo pharmacokinetics and pharmacodynamics of a new triazol, voriconazole in a murine candidiasis model. Antimicrob Agents Chemother 2003; 47: 3165-3169.

2. Andes D, Marchillo K, Conklin R, Krishna G, Ezzet F, Cacciapuoti A Loebenberg D. Pharmacodynamics of a new triazole, posaconazole, in a murine model of disseminated candidiasis. Antimicrob Agents Chemother 2004; 48 : 137-142.

3. Azanza JR, Sádaba B, García Quetglas E, Mensa J. Guía práctica de interacciones de los antibióticos. Madrid, Icono de los antibión y Diseño, 2005.

4. Bafeltowska JJ, Buszman E. Pharmacokinetics of fluconazole in the cerebrospinal fluid of children with hydrocephalus. Chemotherapy 2005; 51 : 370-376.

5. Ballesta S, Garcia I, Perea EJ, Pascual A. Uptake and intracellular activity of voriconazole in human

polymorphonuclear leucocytes. $\mathrm{J}$ Antimicrob Chemother 2005; 55 : 785-787.

6. Capitano B, Potoski BA, Husain S, Zhang S, Paterson DL, Studer SM, McCurry KR, Venkataramanan R. Intrapulmonary penetration of voriconazole in patients receiving an ora prophylactic regimen. Antimicrob Agents Chemother 2006; 50: 1878-1880.

7. Conte JE Jr, Golden JA, Kipps J, Mclver M, Zurlinden E. Intrapulmonary pharmacokinetics and pharmacodynamics of itraconazole and 14-hydroxyitraconazole at steady state. 14-hydroxyitraconazole at steady state. 3823-3827.

8. Courtney R, Pai S, Laughlin M, Lim J, Batra V. Pharmacokinetics, safety, and tolerability of oral posaconazole administered in single and multiple doses in healthy adults. Antimicrob Agents in healthy adults. Antimicrob Agents
Chemother 2003; 47: 2788-2795.

9. Courtney R, Sansone A, Smith W, Marbury T, Statkevich P, Martinho M, Laughlin M, Swan S. Posaconazole pharmacokinetics, safety, and tolerability in subjects with varying degrees of chronic renal disease. J Clin Pharmacol 2005; 45: 185-192.

10. Courtney R, Wexler D, Radwanski E, Lim J, Laughlin M. Effect of food on the relative bioavailability of two ora formulations of posaconazole in healthy adults. Br J Clin Pharmacol 2004; 57 : 218-222.

11. Denes E, Pichon N, Debette-Gratien M, Bouteille B, Gaulier JM. Pharmacokinetics of voriconazole in the cerebrospinal fluid of an immunocompromised patient with a brain abscess due to Aspergillus fumigatus. Clin Infect Dis 2004; 39: 603-604.
12. Elter T, Sieniawski M, Gossmann A Wickenhauser C, Schroder U, Seifert H, Kuchta J, Burhenne J, Riedel KD Fätkenheuer G, Cornely OA. Voriconazole brain tissue levels in rhinocerebral aspergillosis in a successfully treated young woman. Int $\mathrm{J}$ Antimicrob Agents 2006; 28: 262-265.

13. Ezzet F, Wexler D, Courtney R, Krishna G, Lim J, Laughlin M. Oral bioavailability of posaconazole in fasted healthy subjects: comparison between three regimens and basis for clinical dosage recommendations. Clin Pharmacokinet 2005; 44: 211-220.

14. Gubbins PO, Krishna G, Sansone-Parsons A, Penzak SR, Dong L, Martinho M, Anaissie EJ. Martinho M, Anaissie EJ. posaconazole in neutropenic stem cel posaconazole in neutropenic stem cell transplant recipients. Antimicrob A
Chemother 2006; 50: 1993-1999.

15. Hariprasad SM, Mieler WF, Holz ER Gao H, Kim JE, Chi J, Prince RA. Determination of vitreous, aqueous, and plasma concentration of orally administered voriconazole in humans. Arch Ophthalmol 2004; 122: 42-47.

16. Hennig $S$, Wainwright CE, Bell SC, Miller H, Friberg LE, Charles BG. Population pharmacokinetics of itraconazole and its active metabolite hydroxy-itraconazole in paediatric cystic fibrosis and bone marrow transplant patients. Clin Pharmacokinet 2006; 45: 1099-1114.

17. Krieter P, Flannery B, Musick T, Gohdes M, Martinho M, Courtney R. Disposition of posaconazole following single-dose oral administration in healthy subjects. Antimicrob Agents Chemother 2004; 48: 3543-3551.

18. Lohitnavy M, Lohitnavy $\mathrm{O}$, Thangkeattiyanon O, Srichai W. Reduced oral itraconazole bioavailability by antacid suspension. J Clin Pharm Ther 2005; 30: 201-206.

19. Lutsar I, Roffey S, Troke P. Voriconazole concentrations in the cerebrospinal fluid and brain tissue of guinea pigs and and brain tissue of guinea pigs and Dis 2003; 37: 728-732.

20. Mohr JF, Finkel KW, Rex JH, Rodriguez JR, Leitz GJ, Ostrosky-Zeichner L. Pharmacokinetics of intravenous itraconazole in stable hemodialysis patients. Antimicrob Agents hemodialysis patients. Antimicrob

21. Mouton JW, Van Peer A, de Beule K, Van Vliet A, Donnelly JP, Soons PA. Pharmacokinetics of itraconazole and hydroxyitraconazole in healthy subjects after single and multiple doses of a nove formulation. Antimicrob Agents Chemother 2006; 50: 4096-4102.

22. Niwa T, Shiraga T, Takagi A. Drug-drug interaction of antifungal drugs. Yakugaku Zasshi 2005; 125: 795-805.
23. Peng LW, Lien YH. Pharmacokinetics of single, oral-dose voriconazole in peritoneal dialysis patients. Am J Kidney Dis 2005; 45: 162-166.

24. Prentice AG, Glasmacher A. Making sense of itraconazole pharmacokinetics. $\mathrm{J}$ Antimicrob Chemother 2005; 56 (Supl 1): i17-i22.

25. Purkins L, Wood N, Greenhalgh K, Allen MJ, Oliver SD. Voriconazole, a novel wide-spectrum triazole: oral pharmacokinetics and safety. $\mathrm{Br} \mathrm{J}$ Clin Pharmacol 2003; 56 (Supl 1): 10-16.

26. Purkins L, Wood N, Greenhalgh K, Eve MD, Oliver SD, Nichols D. The pharmacokinetics and safety of intravenous voriconazole - a novel widespectrum antifungal agent. Br J Clin

27. Purkins L, Wood N, Kleinermans D, Greenhalgh K, Nichols D. Effect of food on the pharmacokinetics of multiple-dose oral voriconazole. Br J Clin Pharmaco 2003; 56 (Supl 1): 17-23.

28. Saad AH, DePestel DD, Carver PL. Factors influencing the magnitude and clinical significance of drug interaction between azole antifungals and select 2006; 26: 1730-1744.

29. Sadaba B, Campanero MA, Quetglas EG, Azanza JR. Clinical relevance of sirolimus drug interactions in transplant patients. Transplant Proc 2004; 36: 3226-3228.

30. Sansone-Parsons A, Krishna G, Calzetta A, Wexler D, Kantesaria B, Rosenberg MA, Saltzman MA. Effect of a nutritional supplement on posaconazole pharmacokinetics following oral administration to healthy volunteers. Antimicrob Agents Chemother 2006; 50 : 1881-1883.

31. Ullmann AJ, Cornely OA, Burchardt A, Hachem R, Kontoyiannis DP, Töpelt K, Courtney R, Wexler D, Krishna G, Martinho M, Corcoran G, Raad I. Pharmacokinetics, safety, and efficacy of posaconazole in patients with persistent febrile neutropenia or refractory invasive fungal infection. Antimicrob Agents Chemother 2006; 50: 658-666.

32. Von Mach MA, Burhenne J, Weilemann LS. Accumulation of the solvent vehicle sulphobutylether beta cyclodextrin sodium in critically ill patients treated with intravenous voriconazole under renal replacement therapy. BMC Clin Pharmacol 2006; 6: 6.

33. Walsh TJ, Karlsson MO, Driscoll T, Arguedas AG, Adamson P, Saez-Llorens X, Vora AJ, Arrieta AC Blumer J, Lutsar I, Milligan P, Wood N. Pharmacokinetics and safety of intravenous voriconazole in children afte single- or multiple-dose administration. Antimicrob Agents Chemother 2004; 48 2166-2172. 